Research Paper

\title{
Effects of ECM Protein Mimetics on Adhesion and Proliferation of Chorion Derived Mesenchymal Stem Cells
}

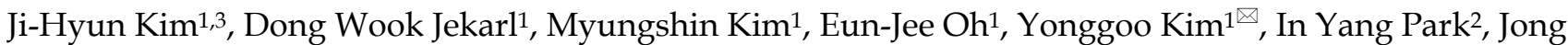
Chul Shin ${ }^{2}$

1. Department of Laboratory Medicine, College of Medicine, The Catholic University of Korea, Seoul, South Korea;

2. Department of Obstetrics and Gynecology, College of Medicine, The Catholic University of Korea, Seoul, South Korea;

3. Department of Biomedical Science, College of Medicine, The Catholic University of Korea, Seoul, South Korea.

$\square$ Corresponding author: Dr. Yonggoo Kim, Department of Laboratory Medicine, The Catholic University of Korea, Seoul St. Mary's Hospital, 505 Seocho-gu, Banpo-dong, Seoul, 137-701, Korea.

(c) Ivyspring International Publisher. This is an open-access article distributed under the terms of the Creative Commons License (http://creativecommons.org/ licenses/by-nc-nd/3.0/). Reproduction is permitted for personal, noncommercial use, provided that the article is in whole, unmodified, and properly cited.

Received: 2013.05.12; Accepted: 2013.08.12; Published: 2014.02.06

\begin{abstract}
Background: We evaluated the effects of fibronectin, collagen, cadherin, and laminin based extracellular matrix (ECM) protein mimetics coated with mussel derived adhesive protein (MAP) on adhesion and proliferation of chorionic mesenchymal stem cells (cMSCs).

Methods: Human placental chorionic tissues from term third-trimester pregnancies $(n=3)$ were used. The CMSCs were cultured on rationally designed ECM protein mimetics coated with MAP on plastic surfaces with the addition of reduced fetal bovine serum $(0.5 \%, \mathrm{I} \% \mathrm{FBS})$. Adhesion capabilities were monitored by a real time cell analysis system (RTCA) utilizing an impedance method. Proliferation capabilities were monitored by RTCA and MTS assay.

Results: Of the ECM protein mimetics tested, GRGDSP(FN) coated surfaces exhibited the highest adhesion and proliferation capabilities on RTCA at FBS concentration of $0.5 \%$ and $1 \%$. When $0.5 \%$ FBS was added to ECM protein mimetics during the MTS assay, GRGDSP(FN), REDV(FN), and collagen mimetics, GPKGAAGEPGKP(Coll) showed higher CMSCs proliferation compared with the control. When I\% FBS was added, GRGDSP(FN) and TAIPSCPEGTVPLYS(CollV) showed significant cMSCs proliferation capacity.

Conclusions: Fibronectin mimetics, GRGDSP(FN) amino acid sequence showed the highest adhesion and proliferation capabilities. In addition, results from RTCA assessment of cell viability correlated well with the tetrazolium-based MTS assay.
\end{abstract}

Key words: chorionic mesenchymal stem cell, extracellular matrix, protein mimetics, real time cell analysis, cell proliferation assay.

\section{Introduction}

Stem cells are capable of both self-renewal and differentiation into diverse types of mature cells [1]. These characteristic are associated with organogenesis and tissue regeneration, which has attracted widespread interest from clinical researchers [2-4]. Among the various types of stem cells, mesenchymal stem cells (MSCs) represent a class of progenitor cells ca- pable of differentiation to several mesenchymal lineages including osteoblasts, adipocytes, and chondroblasts. Bone marrow (BM) is the primary source of MSCs for both experimental and clinical studies, though acquiring these cells carries a number risks such as complications of general anesthesia, pain, risk of viral contamination, and decreasing numbers of 
MSCs with age [5,6]. In addition, MSCs derived from $\mathrm{BM}$ have relatively limited proliferative potential. MSCs derived from placental tissue, on the other hand, may possess greater differentiation capability and proliferative potential, and thus have more recently been used to overcome the limitations of MSCs obtained from BM. MSCs can be harvested from various tissues found in the placenta including the amnion, amniotic fluid, chorion, cord blood, and umbilical cord tissue (Wharton's Jelly). Since the placenta is typically discarded as medical waste after delivery, these tissues can be procured without the use of invasive procedures. In addition, unlike embryonic stem cells, the use of MSCs is less ethically controversial $[4,7]$.

MSCs are adherent to plastic surfaces and interact with individual extracellular matrix (ECM) molecules during attachment, growth, migration, and apoptosis [8]. ECM is the extracellular portion of animal tissue that provides structural support to cells in addition to performing a multitude of other important functions. ECM consists of fibronectin (FN), collagens, laminins, and a variety of other molecules [9]. Fibronectin, a large glycoprotein found in all vertebrates, is involved in cell adhesion, growth, migration, and differentiation $[10,11]$. Collagen is a significant constituent of the natural ECM which promotes the attachment and growth of cells and tissues [12]. Laminin is associated with cell differentiation, migration, and adhesion, as well as phenotype and survival [13]. Therefore, understanding the characteristics of ECM components and the interaction between the ECM and MSCs is important for understanding differentiation and proliferation of stem cells. Full-length ECM components contain adhesive motifs composed of short amino acid sequences that interact specifically with integrins. Unlike full length ECM components, however, the application of short peptides is feasible for surface modification to stimulate differentiation and proliferation of MSCs. The advantages of using short amino acid sequences include increased stability during surface modification and lower production costs $[14,15]$.

Mussels secrete a substance called mussel adhesive protein (MAP) that aids in attachment to surfaces upon which they reside [16]. MAP allows for adherence to wet surfaces, and the adhesion strength is sufficient to secure mussels in place in a rough marine environment [17]. MAP is a polyphenolic protein rich in 3,4-dihydroxyphenylalanine (DOPA) and lysine, which is non-toxic to the human body, biodegradable, and is non-immunogenic [18-20]. These properties make MAP highly attractive for use in a variety of medical, biological, and industrial applications [21]. In cell culture, the strong adhesion ability facilitates effi- cient coating of ECM components on surfaces without protein or surface modifications. ECM protein components can then efficiently promote cellular processes including adhesion, spread, proliferation, differentiation, and survival [8].

For in vitro cell expansion, ECM components of fetal bovine serum (FBS) as well as other materials of animal origin are required for cell culture. Because of critical safety issues, use of animal-derived reagents in clinical applications is not ideal [22,23]. Therefore animal serum-free culture conditions have been developed which use ECM protein mimetics comprised of short peptides. One adhesion-promoting peptide that is commonly used is RGD, which is composed of a tri-amino acid sequence (Arg-Gly-Asp) [24]. Many types of biomaterials have been modified using RGD, and in vitro studies have consistently suggested that RGD-modified surfaces promote better cell attachment compared to that of unmodified surfaces [25].

Recently, ECM protein mimetics comprised of short amino acid sequences attached to plastic surfaces with the aid of MAP have been developed. In this study, we evaluated the use of this tool in culturing placenta-derived chorionic MSCs with various ECM protein mimetics. In addition, by utilizing a real time cell analysis system with real time monitoring of cell viability, the adhesion and proliferation capabilities of placenta derived cMSCs were measured.

\section{Materials and Methods}

\section{Ethics statement}

Human term third trimester placentas were obtained after receiving written informed consent. All experiments were in accordance with the institutional review board guidelines at our medical center (IRB No. KC09WZZZ0173).

\section{Isolation of MSCs from the chorion and culture conditions [26]}

Human chorion $(n=3)$ was obtained from placentas from term third-trimester pregnancies following delivery at Seoul St. Mary's Hospital. The chorionic tissue was washed in Dulbecco's phosphate-buffered saline (DPBS; Gibco, Grand Island, NY, USA) and cut into small pieces $(2 \times 2 \mathrm{~cm})$. The tissue was then incubated with $0.3 \%$ collagenase type I (Gibco) at $37{ }^{\circ} \mathrm{C}$ for $15-30 \mathrm{~min}$. The digested tissue was subsequently passed through a $100 \mu \mathrm{m}$ cell strainer (BD Falcon, Bedford, MA, USA) and the filtered cells were collected by centrifugation at 2500 $\mathrm{rpm}$ for $5 \mathrm{~min}$. The cells were resuspended in a-modified minimum essential medium (a-MEM; Gibco), supplemented with $16.5 \%$ fetal bovine serum (FBS; Hyclone, Logan, UT, USA) and 1\% penicil- 
lin-streptomycin (Gibco, Grand Island, NY, USA), and cultured in T25 flasks (Nunc, Roskilde, Denmark) at $37{ }^{\circ} \mathrm{C}$ in $5 \% \mathrm{CO}_{2}$. This medium was changed twice weekly. When the primary cells (passage 0; P0) reached $70 \%$ confluency, they were trypsinized with $0.5 \%$ trypsin-EDTA (Gibco) and resuspended in T75 flasks (SPL, Pocheon, Kyounggi, Korea). The cells were subcultured repeatedly and were deemed ready for experimental use after passage 3 .

\section{ECM protein mimetics and coating of the plastic culture dish}

After passage 3, cultured cMSCs were used for functional studies. Untreated polystyrene culture plates were coated with various ECM protein mimetics using MAP (MAPTrix ${ }^{\circledR}$, Kollodis BioSciences, Malden, MA, USA). The cMSCs were cultured on various ECM protein mimetics as listed in Table 1. Recombinant MAP without ECM protein mimetics was used as a negative control. The coating materials $(0.1 \mathrm{mg} / \mathrm{mL})$ were used at a volume appropriate for a $125 \mathrm{uL} / \mathrm{cm}^{2}$ well area. After incubating for $2 \mathrm{hrs}$ at 37 ${ }^{\circ} \mathrm{C}$, the wells were washed with the same volume of distilled water and serum-free media.

Table I. ECM protein mimetics investigated in this study.

\begin{tabular}{ll}
\hline ECM mimetics & \\
\hline Fibronectin mimetics & $\begin{array}{l}\text { KNNQKSEPLIGRKKT(FN) } \\
\text { SPPRRARVT(FN) } \\
\text { GRGDSP(FN) } \\
\text { REDV(FN) }\end{array}$ \\
Collagen I mimetics & GPKGAAGEPGKP(ColI) \\
Collagen IV mimetics & $\begin{array}{l}\text { GEFYFDLRLKGDK(ColIV) } \\
\text { TAIPSCPEGTVPLYS(ColIV) }\end{array}$ \\
Cadherin mimetics & LFSHAVSSNG(CAD) \\
Laminin mimetics & KAFDITYVRLKF(LAM) \\
& IKVAV(LAM)
\end{tabular}

\section{Immunophenotyping of cMSCs cultured on ECM protein mimetics}

The presence of cMSCs cultured in 16.5\% FBS was confirmed by immunophenotyping. In addition, cells cultured on GRGDSP(FN) coated surfaces were confirmed by immunophenotyping using anti-CD105 PE, CD73 PE, CD90 PE, CD45 PE, CD34 PE, CD11b PE, CD79a PE, HLA-DR, HLA-DP, and HLA-DQ FITC [27]. The cMSCs in complete culture media containing $0.5 \%$ FBS were pipetted onto a GRGDSP(FN) coated $100 \mathrm{~mm}$ culture dish (SPL). When $70 \%$ confluency was reached, all trypsinized cells were diluted to a concentration $1 \times 10^{6}$ cells $/ \mathrm{mL}$ in phosphate-buffered saline (PBS; Gibco). The cells were labeled with $10 \mathrm{uL}$ of antibodies. For negative controls, cells were labeled with $\mathrm{IgG}_{1}$ and $\mathrm{IgG}_{2}$ isotype controls. The samples were fixed using fixation buffer (BD Biosciences) and analyzed by LSR Fortessa (Becton Dickinson, Franklin Lakes, NJ, USA). A total of 12,000 events were acquired and analyzed using WinMDI 2.9 software.

\section{Differentiation and immunocytochemical staining}

The cMSCs in complete culture media containing $0.5 \%$ FBS were pipetted onto a GRGDSP(FN) coated $100 \mathrm{~mm}$ culture dish (SPL). When $70 \%$ confluency was reached, all trypsinized cells were pipetted onto a 6-well culture plate (Nunc, Roskilde, Denmark) at a concentration of $1 \times 10^{5}$ cells/well. When $70 \%$ confluency was reached in the growth medium, the cells were placed in a differentiation medium and incubated at $37^{\circ} \mathrm{C}$ for $21 \mathrm{~d}$. The differentiation medium was replaced twice weekly. Osteogenic differentiation was performed according to the manufacturer's instructions using an Osteogenesis Differentiation Kit (Gibco), and calcium deposits were subsequently visualized using the Von Kossa Method. Cells were fixed with $4 \%$ paraformaldehyde, placed in a $3 \%$ silver nitrate solution, and exposed to UV light for $60 \mathrm{~min}$. After rinsing with distilled water 3 times, they were then placed in 5\% sodium thiosulfate for $2 \mathrm{~min}$.

Chondrogenic differentiation was performed according to the manufacturer's instructions using a Chondrogenesis Differentiation Kit (Gibco). Chondrogenic differentiation was visualized by measuring metachromatic matrix using Alcian blue staining. Cells were fixed with $4 \%$ paraformaldehyde and placed in 1\% Alcian blue solution for $30 \mathrm{~min}$ at room temperature. After rinsing twice with distilled water, the nuclei were stained using Nuclear Fast Red for 5 $\min$.

\section{Measurement of cell adhesion and proliferation capacity via real time cell analyzer}

The real time cell analyzer and E-plate 16 (RTCA; xCELLigence, Roche, San Diego, CA, USA) system allows for label-free, real time, noninvasive monitoring of the viability of cultured cells using impedance [28]. This platform uses gold electrodes at the bottom of microplate wells as sensors to which an alternating current is applied. Cells are adherently grown in monolayers on top of the electrodes, which influences the alternating current at the electrodes by changing the electrical impedance (denoted as cell index). The cell index values are proportionate to the area of cells 
attached and the total number of cells, which are determined by cell proliferation, cytotoxicity, cytoprotection, cell growth inhibition, and apoptosis [29-33]. The cell index is a relative values calculated by analyzer, the cell index values of control was used as baseline values of experiments. The cell index values divided by control were utilized as cell index ratio.

The effects of ECM protein mimetics on cMSCs adhesion and proliferation were monitored using RTCA as previously reported [34]. A total of $50 \mathrm{uL}$ of cell culture media was added to various ECM protein mimetic coated E-plates. After incubation at room temperature for $15 \mathrm{~min}$, the background intensity was measured. Adhesion capacity of cMSCs, which was visualized in increments of the cell index, was determined as per the manufacturer's instructions. In brief, 15,000 cells were suspended in $100 \mathrm{uL}$ of serum-reduced culture medium $(0,0.1,1,5,10,16.5 \%$ FBS) and seeded into each well. Immediately, an E-plate was installed into the RTCA system, and the cell index was measured every minute. To evaluate proliferation capacity, 5,000 cMSCs were suspended in $100 \mathrm{uL}$ of serum-reduced culture medium $(0.5 \%$, $1 \%, 5 \%$ FBS) and seeded into each well. Immediately, an E-plate was installed into the RTCA system, and the cell index was measured every $10 \mathrm{~min}$.

The cell proliferation capabilities of cMSCs on ECM protein mimetics were measured using a CellTiter 96 Aqueous One Solution Cell Proliferation Assay Kit (Promega, Madison, WI, USA). A total of

\section{Determining cell proliferation using the MTS assay}

5,000 cells per well were seeded onto various ECM protein mimetics coated onto a 96-well cell culture plate (SPL). After $72 \mathrm{hrs}$, a tetrazolium compound [3-(4,5-dimethylthiazol-2-yl)-5-(3-carboxymethoxyphe nyl)-2-(4-sulfophenyl)-2H-tetrazolium, tinner salt] which was bioreduced by cells into a formazan product, was added and incubated at $37^{\circ} \mathrm{C}$ for 2-3 hrs. The amount of formazan product, which was proportional to the number of living cells, was measured at an absorbance of 490nm by an Absorbance Microplate Reader (VersaMax; Molecular Devices, USA). The proliferation rates of the cells were calculated using the absorbance of cMSCs cultured on various ECM protein mimetics divided by the absorbance of control MSCs. These rates are expressed as a ratio.

\section{Results}

\section{Characteristics of human cMSCs}

The cMSCs $(n=3)$ attached and proliferated on plastic tissue culture plates, and subsequently presented with a fibroblast-like morphology 7 days after seeding. As the passage number was increased, the cMSCs exhibited a typical triangular shape that became elongated and thinner compared to the first day after seeding. The morphology of cMSCs was observed until passage 3 , at which time they were used in the experiment (Fig. 1). The cMSCs which were cultured in $16.5 \%$ FBS on a GRGDSP(FN) coated surface, showed that $\geq 95 \%$ of the population expressed CD105, CD73, and CD90, and $\leq 2 \%$ of the population expressed CD45, CD34, CD11b, CD79a, or HLA-DR (Fig. 2).

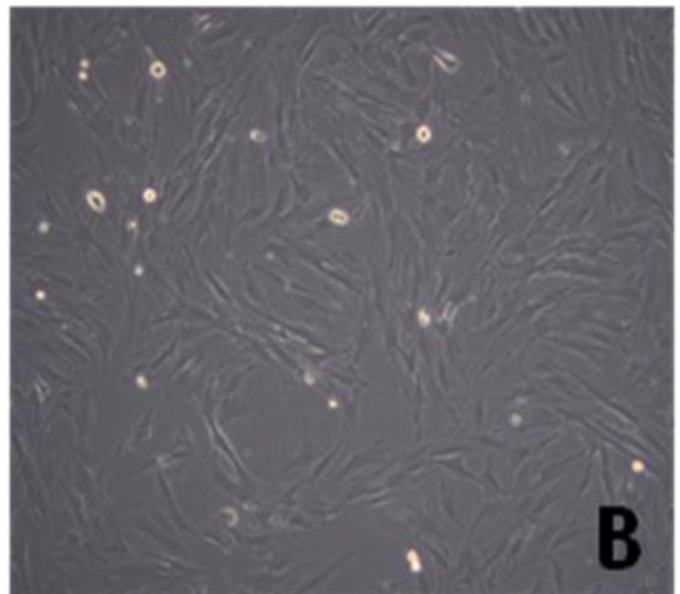

Figure I. Morphology of human chorionic mesenchymal stem cells after passage 3. Cells were observed to have the typical morphology of fibroblast-like cells on phase contrast microscopy (A: magnification=50x, B:100x). 

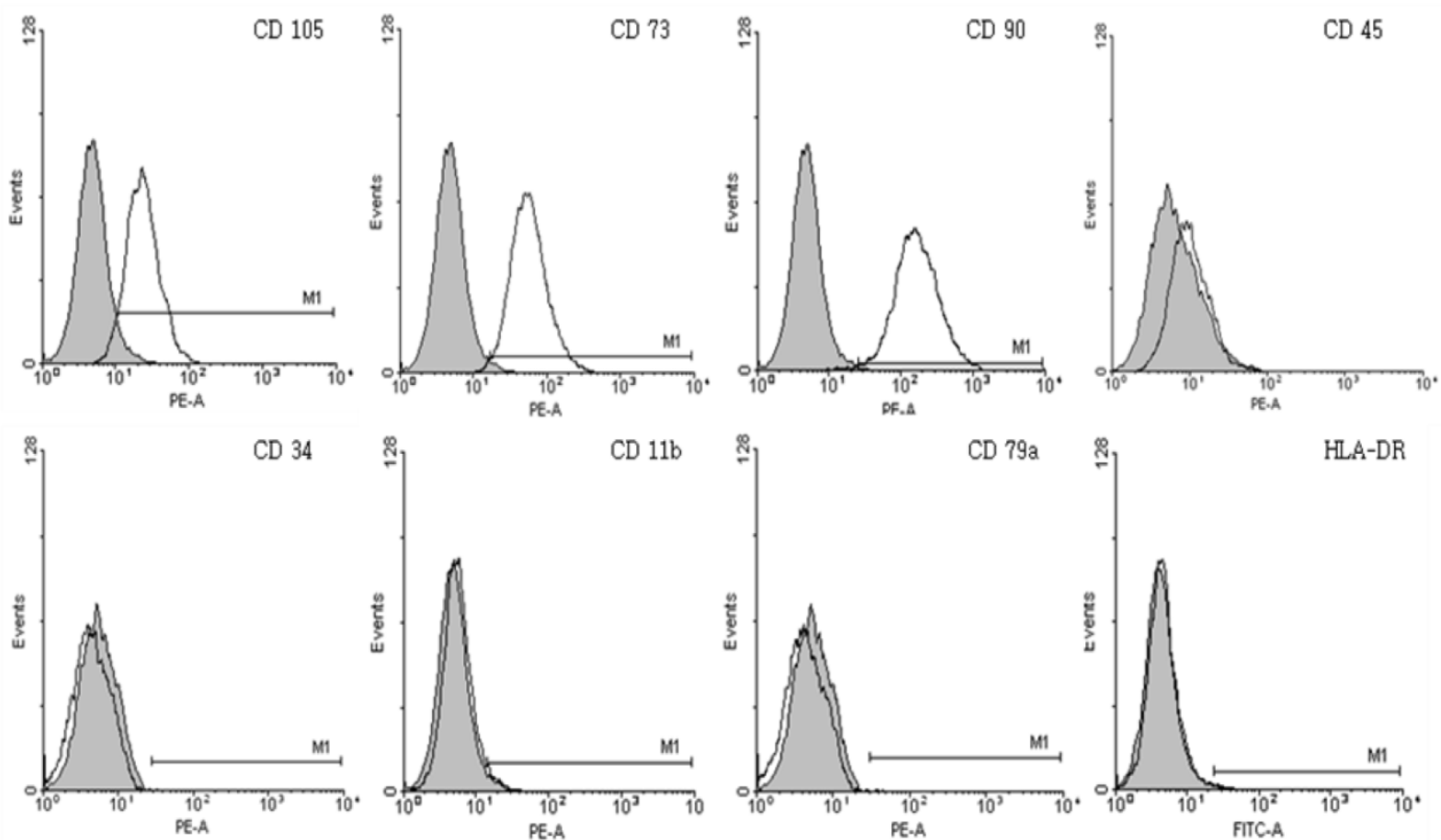

Figure 2. Immunophenotypic characterization of chorionic mesenchymal stem cells. The expression of surface antigens was confirmed by immunophenotyping. Cultured chorionic mesenchymal stem cells on GRGDSP(FN) coated surfaces were positive for typical mesenchymal stem cell surface markers including CDI05, CD73 and CD90, but not hematopoietic markers including CD45, CD34, CD79a, or HLA-DR. Colored histogram: isotype control. Open histogram: signal for each specific Ab.

\section{Differentiation of cMSCs}

Standard differentiation assays were performed to confirm stemness of cMSCs. Cells cultured in 16.5\% FBS on a GRGDSP(FN) coated surface differentiated into osteoblasts and chondroblasts. After 3 weeks of culture in osteogenic induction medium, cMSCs cultured under both conditions were able to undergo osteogenic differentiation as demonstrated by the development of positive Von Kossa staining. Dark-brown calcium deposits were also observed using silver nitrate solution (Fig. 3A). Likewise, after 3 weeks of culture in chondrogenic induction medium, cMSCs were able to undergo chondrogenic differentiation as demonstrated by the development of positive Alcian blue staining. In these cases, the metachromatic matrix was stained blue in color, and nuclei were stained red by Nuclear Fast Red stain (Fig. 3B).

\section{Assessment of adhesion of cMSCs to ECM protein mimetic coated surfaces using RTCA}

The effects of FBS on proliferation of cMSCs were evaluated in this study. The addition of $0 \%$ and $0.1 \%$ FBS concentrations to the medium elicited no change in cell index values compared to the initial value, which indicates growth failure. FBS concentrations of $1.0 \%, 5.0 \%, 10.0 \%$, and $16.5 \%$ induced proportionate changes in the cell index (Fig. 4). The adhesion capabilities of cMSCs on surfaces coated with various ECM protein mimetics were observed for 3 hrs. We found that cMSCs neither attached nor proliferated in $0 \%$ FBS on ECM protein mimetics. cMSCs attached, but did not proliferate, in media containing $0.1 \%$ FBS along with any of the tested ECM protein mimetic coated surfaces. The cell indices of cMSCs in media containing $0.1 \%$ FBS along with various ECM protein mimetic coated surfaces were higher than those containing MAP (without mimetics) coated surfaces, which were regarded as the negative control. As a result, GRGDSP (FN) coated surface provided the best condition for cell adhesion (Figure 5).

\section{Assessment of proliferation of cMSCs on ECM protein mimetic coated surfaces using RTCA}

Chorionic MSC proliferation on ECM protein mimetic coated surfaces was studied for 72 hrs using RTCA. Proliferation assays were carried out under serum-reduced conditions to evaluate the effects of ECM protein mimetics. cMSCs were cultured in two groups of media, each of which contained a low concentration of FBS $(0.5 \%, 1 \%)$. The cMSCs in $0.5 \%$ and $1 \%$ without protein mimetics were regarded as baseline control. In 0\% FBS along with ECM protein mimetics, cMSC proliferation was not observed. In $0.5 \%$ FBS, all ECM protein mimetics showed an increased cell index ratio, which is indicative of cMSCs proliferation capacity. Among them, cMSCs cultured on GRGDSP(FN) coated surfaces showed the highest cell index which was $3.49,3.13,2.71,2.28,2.12$ and 2.19 at 
9, 16, 24, 36, 48 and 72 hour respectively (Figure 6A, $\mathrm{C} 1, \mathrm{C} 2)$. With the addition of $1 \%$ FBS, all the ECM mimetics except for IKVAV (LAM) supported greater MSC proliferation compared to the control (Figure 6B, D1, D2). From 48 hrs, IKVAV (LAM) showed lower

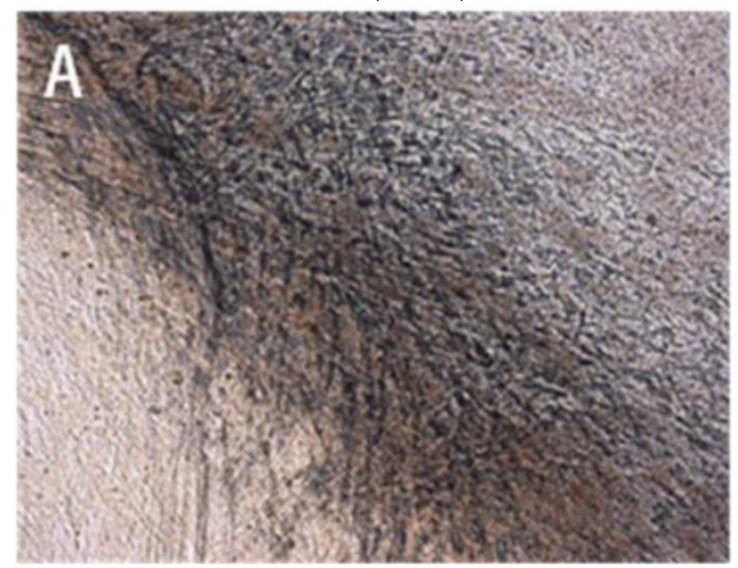

cell index ratio compared to control. Regardless of different FBS concentration, cMSCs cultured on GRGDSP (FN) coated surfaces showed the greatest proliferation capability.

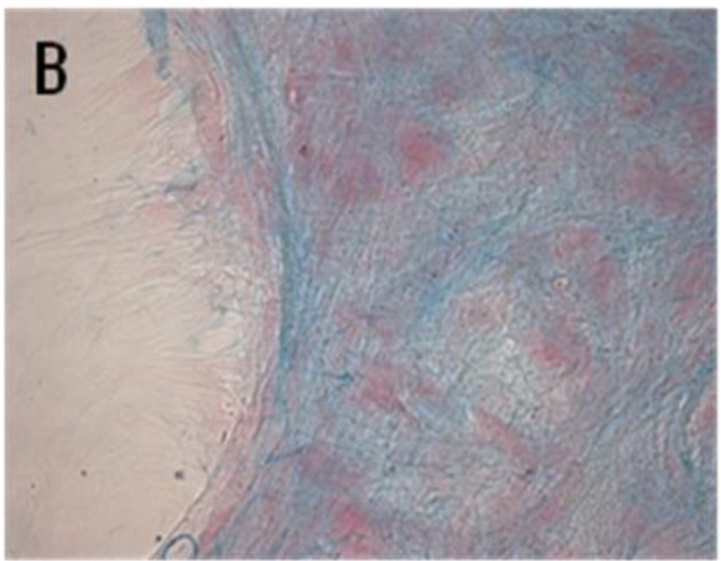

Figure 3. cMSCs incubated in osteogenic and chondrogenic differentiation media for $2 \mathrm{I}$ days. (A) Osteogenic differentiation was detected immunologically by von Kossa staining and (B) chondrogenic differentiation was detected by Alcian blue staining (x200).
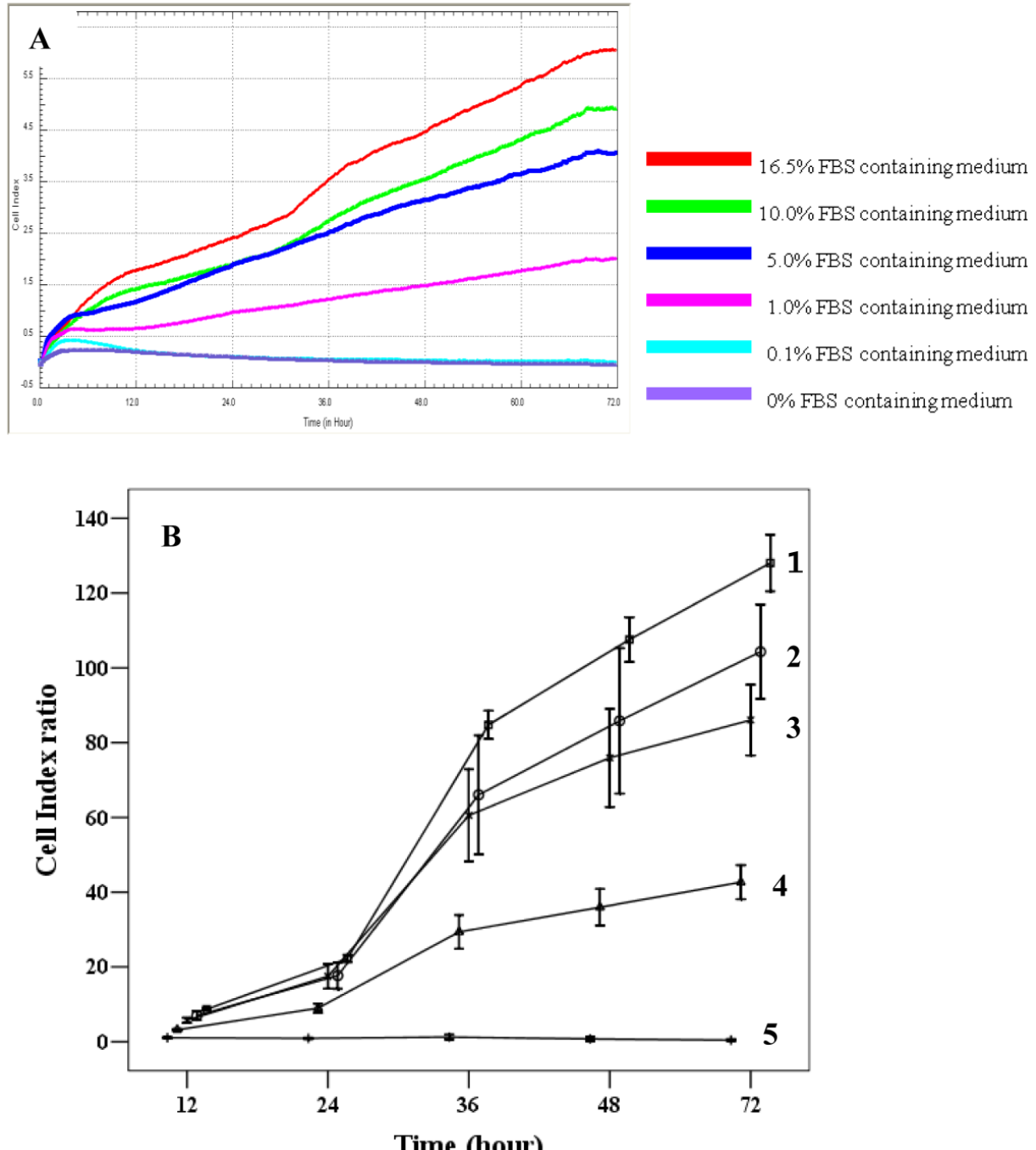

Figure 4. Proliferation capacity of chorionic MSCs in various FBS concentrations continuously monitored by RTCA for 72 hrs (A) and plotted by cell index ratio which was obtained by value divided by control cell index (B). cMSCs in $0.1 \%$ to $16.5 \%$ FBS are shown. Abbreviations: $1,16.5 \%$ FBS, $2 ; 10.0 \%$ FBS; $3,5.0 \%$ FBS; $4,1.0 \% \mathrm{FBS} ; 5,0.1 \%$ FBS. 

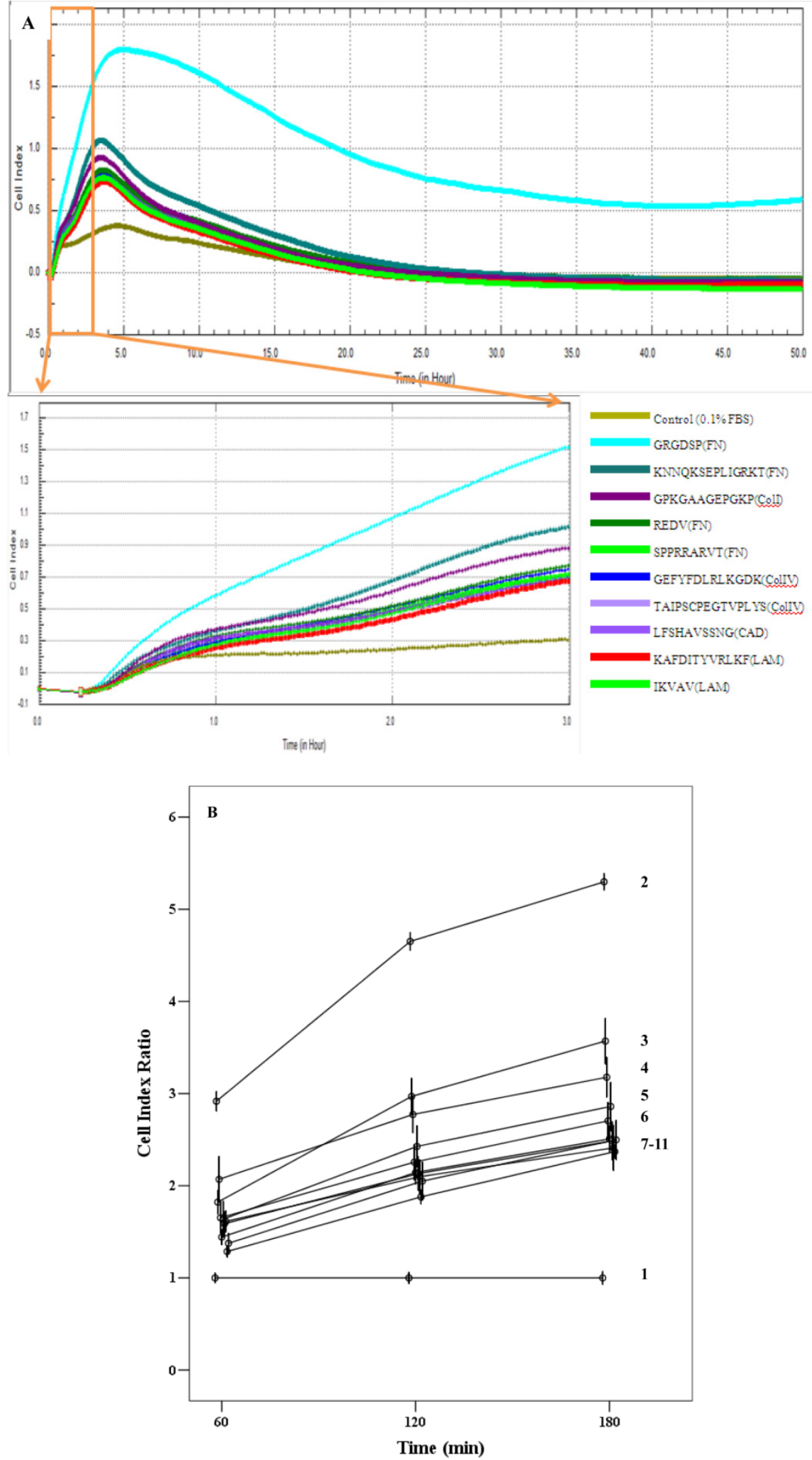

Figure 5. Adhesion capacity of MSCs in $0.1 \%$ FBS containing medium. Cell index of cMSCs in $0.1 \%$ FBS with ECM protein mimetic coated surfaces are monitored by RTCA (A) and plotted by cell index ratio which was obtained by value divided by control cell index (B). The cMSCs in $0.1 \%$ FBS were regarded as the baseline control group. Abbreviations: I, Control (0.1\% FBS); 2, GRGDSP(FN); 3, KNNQKSEPLIGRKT(FN); 4, GPKGAAGEPGKP (Coll); 5, REDV (FN); 6, GEFYFDLRLKGDK (CollV); 7, SPPRRARVT(FN); 8, TAIPSCPEGTVPLYS(ColIV); 9, IKVAV(LAM); I0, LFSHAVSSNG (CAD); II, KAFDITYVRLKF(LAM). 

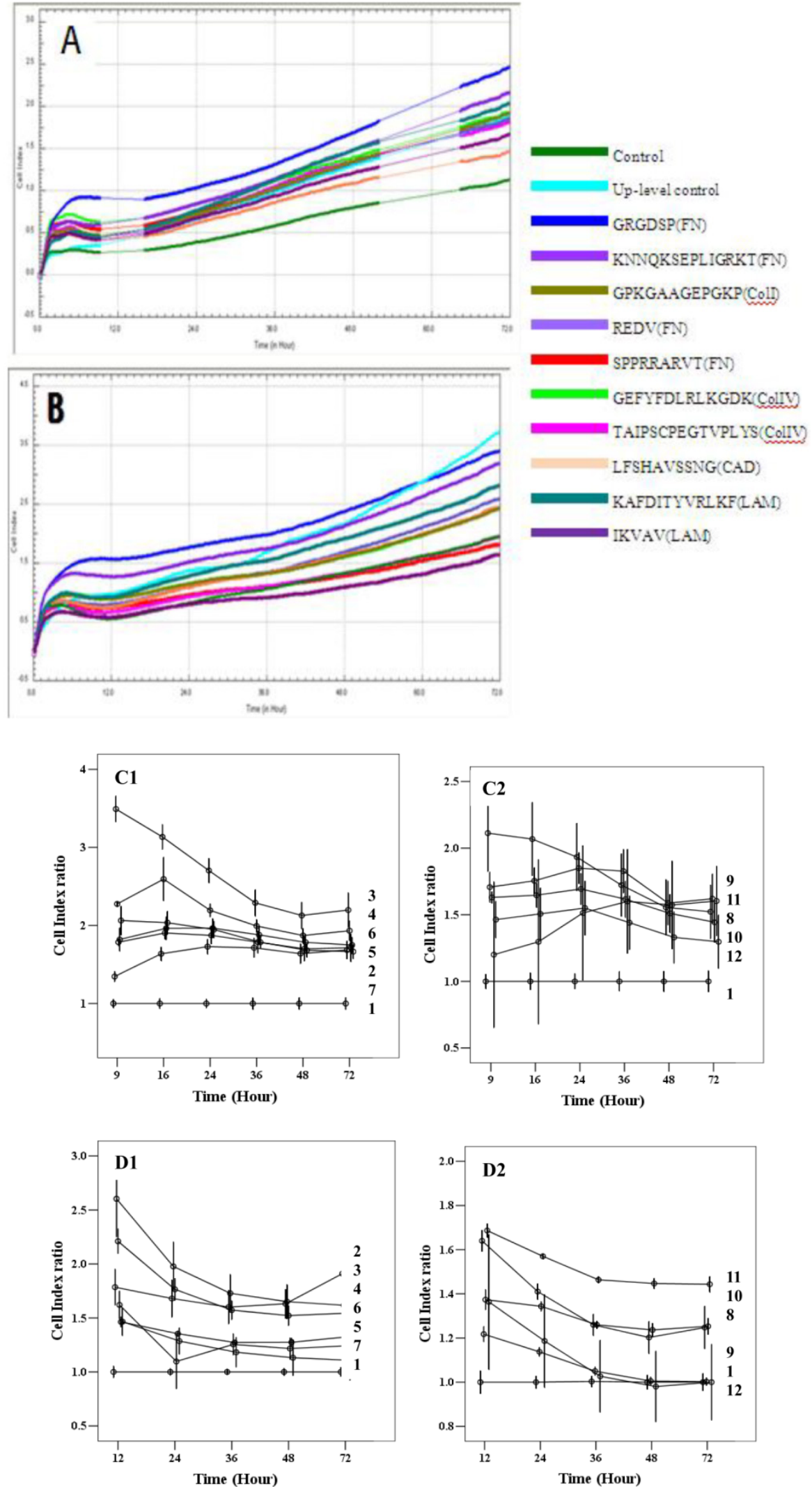

Figure 6. Cell index of cMSCs proliferation in (A) $0.5 \%$ FBS, (B) I\% FBS. Impedances for each group are shown as follows: baseline control group, green; up-level control group, cyan; GRGDSP(FN), blue; KNNQKSEPLIGRKKT(FN), blue violet; GPKGAAGEPGKP(Coll), olive; REDV(FN), slate blue; SPPRRARVT(FN), red; GEFYFDLRLKGDK(CollV), yellow-green; TAIPSCPE-GTVPLYS(CollV), magenta; LFSHAVSSNG(CAD), coral; KAFDITYVRLKF(LAM), dark cyan; IKVAV(LAM), dark magenta. Impedance profiles were recorded in quadruplicate continuously over 72 hrs using the RTCA system (A, B) and plotted by cell index ratio which was obtained by value divided by control cell index (C, $0.5 \% \mathrm{FBS} ; \mathrm{D}, 1 \% \mathrm{FBS})$. Abbreviations: I, Control (0.1\% FBS); 2, Up level control; 3, GRGDSP(FN); 4, KNNQKSEPLIGRKT(FN); 5, GPKGAAGEPGKP(Coll); 6, REDV(FN); 7, SPPRRARVT(FN); 8, GEFYFDLRLKGDK (CollV); 9, TAIPSCPEGTVPLYS(ColIV); I0, LFSHAVSSNG (CAD); II, KAFDITYVRLKF(LAM); I2, IKVAV(LAM). 


\section{Assessment of proliferation of cMSCs on ECM protein mimetic coated surfaces using an MTS assay}

The cMSCs proliferation capabilities on ECM protein mimetic coated surfaces were investigated using an MTS assay. When $0 \%$ FBS was added to ECM protein mimetics, cMSCs proliferation was not observed. After $0.5 \%$ FBS was added to ECM protein mimetics, GRGDSP(FN), REDV(FN), and GPKGAAGEPGKP(ColI) showed higher cMSCs proliferation compared to the control [GRGDSP(FN), $119 \% \pm 13.12 \%, p=0.008 ; \operatorname{REDV}(\mathrm{FN}), 118 \% \pm 17.19 \%$, $p=0.020 ; \quad$ GPKGAAGEPGKP(ColI), $114 \% \pm 11.44 \%$, $p=0.013$ ) (Fig. 7A). After 1\% FBS was added, GRGDSP (FN) and TAIPSCPEGTVPLYS (ColIV) showed significantly higher ratios compared to the control [GRGDSP (FN), $112 \% \pm 9.10 \%, \quad p=0.013$; TAIPSCPEGTVPLYS (ColIV), 109\% $\pm 9.78 \%, p=0.029$ ) (Fig. 7B). After 5\% FBS was added to the ECM protein mimetics, the proliferation capacity was not significantly different from that of the control (Fig. 7C).

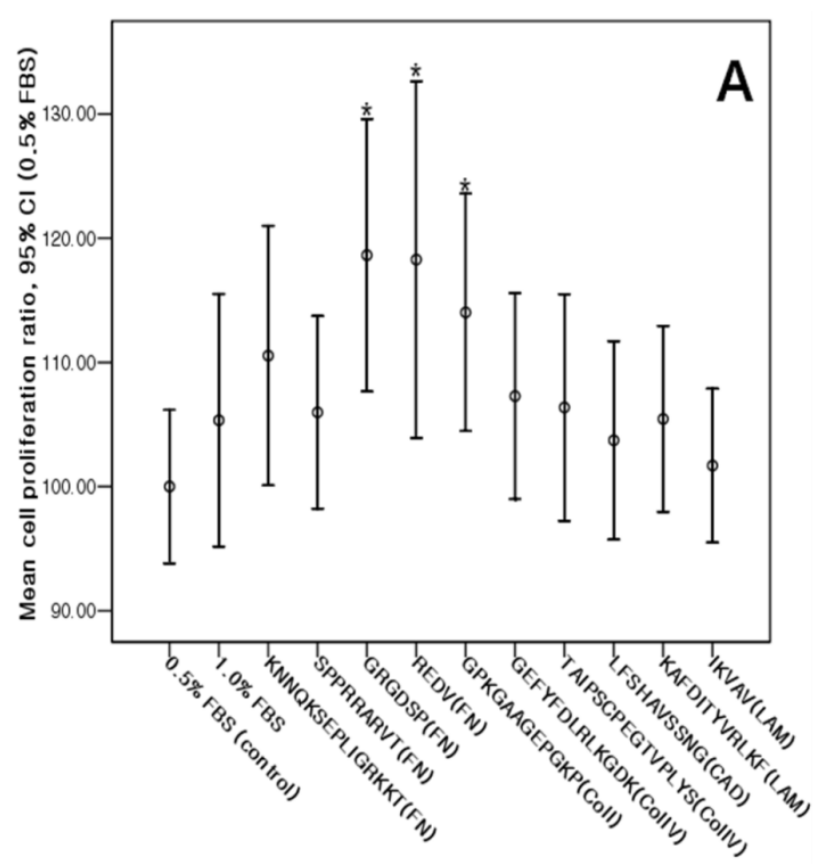

\section{Discussion}

The human placenta, which is typically discarded after delivery, has emerged as a source of MSCs with greater differentiation capacity than that of adult bone marrow derived MSCs $[4,35,36]$. The placenta is an important source of progenitor cells including amniotic epithelial, amniotic mesenchymal, chorionic mesenchymal and chorionic trophoblastic cells [4]. Of these, MSCs derived from chorionic
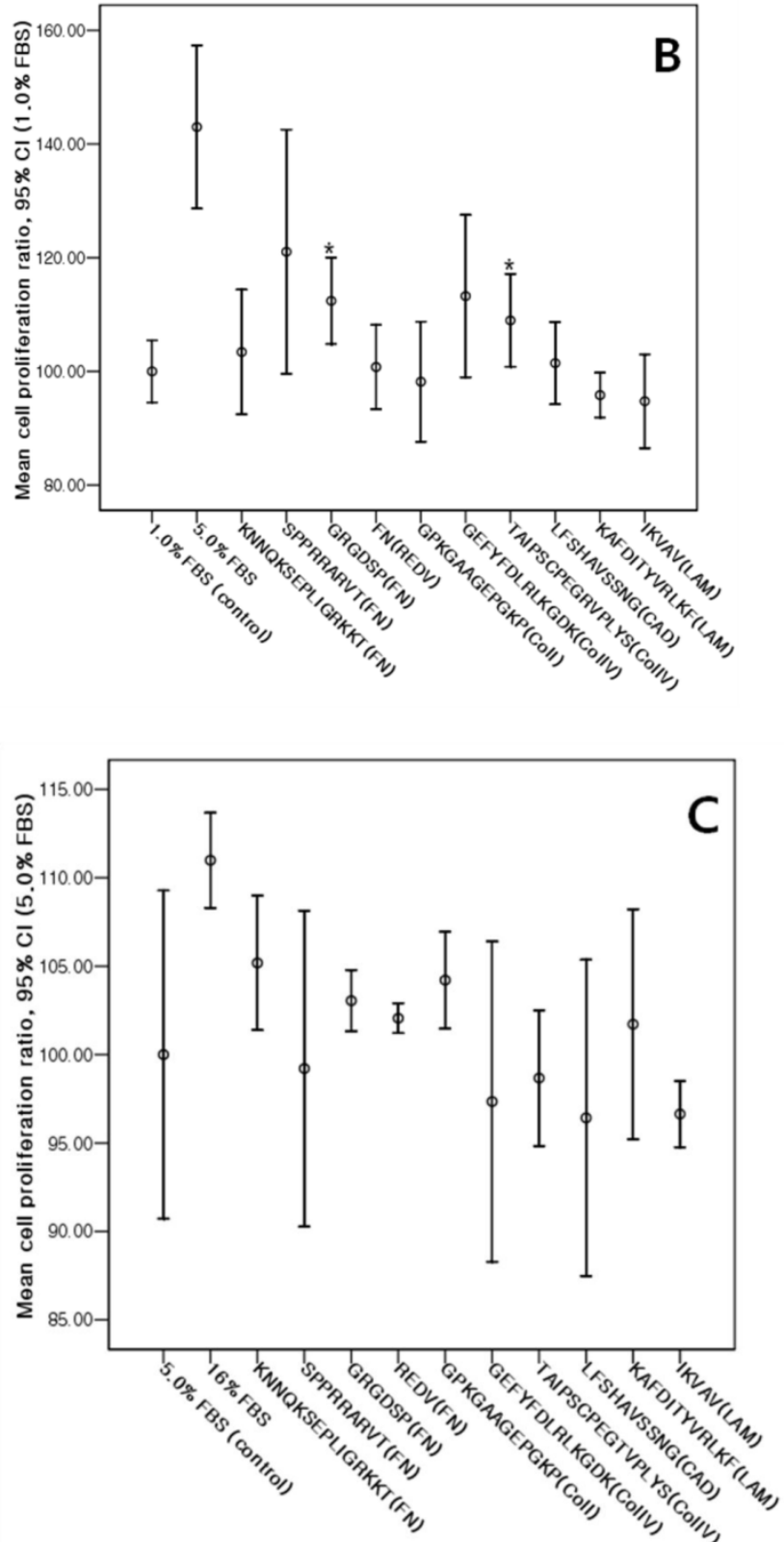

Figure 7. Various ECM protein mimetics used for $\mathrm{CMSCs}$ proliferation. CMSCs were seeded on ECM protein mimetic coated 96-well plates and cultured for $72 \mathrm{hrs}$ in a culture medium containing (A) $0.5 \% \mathrm{FBS}$, (B) $1 \%$ FBS, or (C) $5 \%$ FBS. The cells were quantified by MTS assay. Each bar represents the mean value of each quadruplicate. Results are reported as a percentage compared to the control. Means \pm SD are shown; * p-value $<0.05$.

mesenchymal tissue in particular are known to possess expansion and multilineage differentiation capabilities [26].

Previous studies have reported that ECM components modulate MSC adhesion, migration, proliferation, and osteogenic differentiation. The utilization of specific short peptides originating from the ECM coated by MAP [8] has been shown to simulate in vivo local micro-environments which affect cell attachment, migration, proliferation, differentiation, and 
secretion of factors [14]. Among these sequences, the RGD peptide is composed of a tri-amino acid sequence (Arg-Gly-Asp) and is one of the most widely-studied adhesion-promoting peptides [24]. Many types of biomaterials have been modified using RGD, and in vitro studies consistently suggest that RGD-modified surfaces promote better cell attachment compared to that of unmodified surfaces [25,37]. For this study, short ECM protein mimetics were designed and coated with MAP on plastic surfaces.

Among the ECM protein mimetics studied, GRGDSP(FN) coated surfaces showed the highest cell index ratio and mean cell proliferation ratio. Therefore, cMSCs expanded on GRGDSP(FN) coated surfaces were tested for immunophenotype and differentiation capability. These cMSCs were positive for CD105, CD73, and CD90, but negative for CD45, CD34, CD11b, CD79a, and HLA-DR, which is similar to that seen in BM MSCs. Low HLA-DR expression in particular indicates that cMSCs have an immune-privileged status, and that their immunosuppressive effects may allow for use as a source of stem cells for transplantation [7,38,39]. The multilineage capability of human cMSCs was shown by successful osteogenic and chondrogenic differentiation.

Since the number of MSCs available from BM or placentas is small, MSC expansion in vivo is necessary for clinical applications. In vivo culture of MSCs requires the addition of FBS in short term culture protocols, which is known to stimulate immune reactions. Therefore, FBS-free or reduced methods are ideal [40]. Previous studies have described an FBS-free method using fresh frozen plasma/plasma with platelet lysate [41]. The use of ECM protein mimetics could be an alternative to using FBS, as shown in this study. However, ECM protein mimetics by themselves failed to support the growth of MSCs, as a minimum of $0.5 \%$ FBS was required for growth. Further studies will be necessary to explore the possibility of substituting platelet lysate, FFP, or another medium for $0.5 \%$ FBS.

Monitoring growth and viability of cells without labeling was performed using the RTCA xCELLigence system. The impedance measurements, which are displayed as cell index values, provide quantitative information about the biological status of the cells including cell number, viability, and morphology [28]. Continuous monitoring of proliferation of cells and presentation of result by real time could enhance analytical accuracy. The cell index is known to correlate well with cell number and WST-1, which is a tetrazolium salt used for measurement of cell viability [28]. In this study, results from xCELLigence and MTS assays also showed correlations. After the addition of $0.5 \%$, all ECM protein mimetics showed higher growth kinetics compared to the control, with the highest cell index belonging to GRGDSP(FN). Likewise in the MTS assay, the mean proliferation ratio of ECM protein mimetics was higher compared to the control, with GRGDSP(FN) showing the highest ratio. In this study, fibronectin-based ECM protein mimetics exhibited the highest proliferation ratio on MTS assays. The p-values from comparisons between ECM protein mimetics including fibronectin, cadherin, and laminin along with $0.5 \%, 1.0 \%$ or $5.0 \%$ FBS were $p=0.02, p=0.006$, and $p=0.01$, respectively. Fibronectin based ECM protein mimetics showed higher proliferation rates than other protein mimetics. Unexpectedly, cadherin and laminin based ECM protein mimetics in $0.5 \%$ and $5.0 \%$ FBS conditions showed similar or lower ratios compared to the control, which may indicate a suppressive effect. However, Previous literatures reported that the adhesion of MSC cultured at $0.1 \%$ fetal calf serum (FCS) and proliferation capability cultured at 1\% FCS with different ECM components showed that the laminin-1 was the lowest capabilities compared to collagen and fibronectin [14]. Others reported that colony forming efficacy of MSC at $20 \%$ FBS with vitronectin, fibronectin, laminin showed increased capability [42]. Unlike RGD peptide containing fibronectin, effect of laminin on MSC adhesion and proliferation was incoherent depending on culture condition $[14,42]$ and different amino acid sequence as in this study.

Since ECM protein mimetics coated by MAP supported the growth of cMSCs, these strategies could possibly be applied to clinical therapy. Mussel protein is known to be non-toxic to the human body and does not impose immunogenicity [17]. Therefore, ECM protein mimetics coated by MAP could support the local healing process on prostheses or large bone defects by facilitating growth and differentiation of MSCs.

\section{Conclusion}

Of the ECM protein mimetics used in this study, fibronectin protein mimetics, GRGDSP(FN) coated with MAP elicited the greatest adhesion and proliferation capabilities of MSCs. In addition, data regarding cell viability obtained using the real time cell analysis system correlated well with the Tetrazolium based MTS assay.

\section{Acknowledgement}

This study was supported by a grant from the Korea Healthcare technology R\&D Project, Ministry for Health, Welfare \& Family Affairs, Republic of Korea (SN: A092258). 


\section{Competing Interests}

The authors have declared that no competing interest exists.

\section{References}

1. Kadereit S, Udolph G. Umbilical cord blood. New York: World Scientific Publishing Co. 2011

2. Pittenger MF, Mackay AM, Beck SC, et al. Multilineage potential of adult human mesenchymal stem cells. Science. 1999; 284 :143-7.

3. Song IH. Adult Mesenchymal Stem Cells for Cell Therapy in Clinical Application. Yeungnam Univ. J. of Med. 2009; 26: 1-14.

4. Parolini O, Alviano F, Bagnara GP, et al. Concise review: isolation and characterization of cells from human term placenta: outcome of the first international Workshop on Placenta Derived Stem Cells. Stem Cells. 2008; 26: 300-11.

5. Yoon YS, Jeong HC, Hwang JH, et al. Isolation and characterization of mesenchymal stem cells from human amnion and deciduas. Korean J Obstet Gynecol. 2008; 51: 1269-79.

6. Romanov YA, Svintsitskaya VA, Smirnov VN. Searching for alternative sources of postnatal human mesenchymal stem cells: candidate MSC-like cells from umbilical cord. Stem Cells. 2003; 21: 105-10.

7. Fariha MM, Chua KH, Tan GC, et al. Human chorion-derived stem cells: changes in stem cell properties during serial passage. Cytotherapy. 2011; 13: $582-93$

8. Choi BH, Choi YS, Kang DG, et al. Cell behavior on extracellular matrix mimic materials based on mussel adhesive protein fused with functional peptides. Biomaterials. 2010; 31: 8980-8.

9. Marastoni S, Ligresti G, Lorenzon E, et al. Extracellular matrix: a matter of life and death. Connect Tissue Res. 2008; 49: 203-6.

10. Hynes RO. Integrins: versatility, modulation, and signaling in cell adhesion. Cell. 1992; 69: 11-25.

11. Cukierman E, Pankov R, Yamada KM. Cell interactions with three-dimensional matrices. Curr Opin Cell Biol. 2002; 14: 633-9.

12. O'Brien FJ, Harley BA, Yannas IV, et al. The effect of pore size on cell adhesion in collagen-GAG scaffolds. Biomaterials. 2005; 26: 433-41.

13. Timpl R, Rohde H. Laminin - A Glycoprotein from Basement Membranes. The Journal of Biological Chemistry. 1979; 254: 9933-7.

14. Ode A, Duda GN, Glaeser JD, et al. Toward biomimetic materials in bone regeneration: functional behavior of mesenchymal stem cells on a broad spectrum of extracellular matrix components. J Biomed Mater Res A. 2010; 95: 1114-24

15. Peter $\mathrm{X}$ Ma. Biomimetic Materials for Tissue Engineering. Adv Drug Deliv Rev. 2008; 60: 184-98.

16. Dalsin JL, $\mathrm{Hu} \mathrm{BH}$, Lee BP, et al. Mussel adhesive protein mimetic polymers for the preparation of nonfouling surfaces. J Am Chem Soc. 2003; 125: 4253-8.

17. Hwang DS, Sim SB, Cha HJ. Cell adhesion biomaterial based on mussel adhesive protein fused with RGD peptide. Biomaterials 2007; 28: 4039-46.

18. Dove J, Sheridan P. Adhesive protein from mussels: possibilities for dentistry, medicine, and industry. J Am Dent Assoc. 1986; 112: 879.

19. Green K. Mussel adhesive protein. In: Sierra DH, Saltz RL, editors. Surgical adhesive and sealants. Current technology and application. Lancaster, PA: Technomic, 1995; :19-27.

20. Hwang DS, Yoo HJ, Jun JH, et al. Expression of functional recombinant mussel adhesive protein Mgfp-5 in Escherichia coli. Appl Environ Microbiol. 2004; 70: $3352-9$.

21. [Internet] http://www.kollodis.com/sub0201.htm

22. Lindroos B, Boucher $\mathrm{S}$, Chase $\mathrm{L}$, et al. Serum-free, xeno-free culture media maintain the proliferation rate and multipotentiality of adipose stem cells in vitro. Cytotherapy. 2009; 11: 958-72.

23. Sohn MY, Seo JM, Cho H-J, et al. Effective In Vitro Expansion of Amniotic Fluid-Derived Stem Cells in Serum Free Medium. Tissue Engineering and Regenerative Medicine. 2012; 9: 48-54.

24. Hersel U, Dahmen C, Kessler H. RGD modified polymers: biomaterial for stimulated cell adhesion and beyond. Biomaterials. 2003; 24: 4385-415.

25. Sawyer AA, Hennessy KM, Bellis SL. The effect of adsorbed serum proteins, RGD and proteoglycan-binding peptides on the adhesion of mesenchymal stem cells to hydroxyapatite. Biomaterials. 2007; 28: 383-92.

26. Koo BK, Park IY, Kim J, et al. Isolation and characterization of chorionic mesenchymal stromal cells from human full term placenta. J Korean Med Sci. 2012; 27: 857-63.

27. Dominici M, Blance $\mathrm{K}$, Mueller I, et al. Minimal criteria for defining multipotent mesenchymal stromal cells. The international society for cellular therapy position statement. Cytotherapy. 2006; 8: 315-317

28. Ke N, Wang $X, X u X$, et al. The xCELLigence system for real-time and label-free monitoring of cell viability. Methods Mol Biol. 2011; 740: 33-43.

29. Solly K, Wang X, Xu X, et al. Application of real-time cell electronic sensing (RT-CES) technology to cell-based assays. Assay Drug Dev Technol. 2004; 2: $363-72$

30. Angstmann M, Brinkmann I, Bieback K, et al. Monitoring human mesenchymal stromal cell differentiation by electrochemical impedance sensing. Cytotherapy. 2011; 13: 1074-89.
31. Park HE, Kim D, Koh HS, et al. Real-Time Monitoring of Neural Differentiation of Human Mesenchymal Stem Cells by Electric Cell-Substrate Impedance Sensing. Journal of Biomedicine and Biotechnology. 2011.

32. Ozsvári B, Puskás LG, Nagy LI, Kanizsai let al. A cell-microelectronic sensing technique for the screening of cytoprotective compounds. Int J Mol Med. 2010; 25: 525-30.

33. Label-Free Assay for NK Cell-mediated Cytolysis.

34. Moodley K, Angel CE, Glass M, et al. Real-time profiling of NK cell killing of human astrocytes using xCELLigence technology. J Neurosci Methods. 2011; 200: $173-80$.

35. Zhang X, Mitsuru A, Igura $\mathrm{K}$, et al. Mesenchymal progenitor cells derived from chorionic villi of human placenta for cartilage tissue engineering. Biochem Biophys Res Commun. 2006; 340: 944-52.

36. Yust-Katz S, Fisher-Shoval Y, Barhum Y, et al. Placental mesenchymal stromal cells induced into neurotrophic factor-producing cells protect neuronal cells from hypoxia and oxidative stress. Cytotherapy. 2012; 14: 45-55.

37. Lee GSR, Kim DC, Choi SK, et al. Expression of embryonic stem cell markers in human term placenta. Korean Journal of Obstetrics and Gynecology. 2009; 52: 700-706.

38. Ilancheran S, Moodley Y, Manuelpillai U. Human fetal membranes: a source of stem cells for tissue regeneration and repair? Placenta. 2009; 30: 2-10.

39. Le Blanc K. Immunomodulatory effects of fetal and adult mesenchymal stem cells. Cytotherapy. 2003; 5: 485-9.

40. Horwitz EM, Gordon PL, Koo WK, et al. Isolated allogeneic bone marrow-derived mesenchymal cells engraft and stimulate growth in children with osteogenesis imperfect: Implications for cell therapy of bone. Proc Natl Acad Sci U S A. 2002; 99: 8932-7.

41. Muller I, ordowich S, Holzwarth C, et al. Animal serum-free culture conditions for isolation and expansion of multipotent mesenchymal stromal cells from human BM. Cytotherapy. 2006; 8: 437-44.

42. Gronthos S, P Simmons, Graves S, et al. Integrin-mediated interactions between human bone marrow stromal precursor cells and the extracellular matrix. Bone. 2001; 28: 174-181. 